Théologiques

Théologiques

\title{
La théologie dans le champ spéculatif
}

\section{Jean-François Malherbe}

Volume 1, numéro 1, mars 1993

Théologie et sciences humaines en contexte universitaire

URI : https://id.erudit.org/iderudit/602384ar

DOI : https://doi.org/10.7202/602384ar

Aller au sommaire du numéro

\section{Éditeur(s)}

Faculté de théologie de l'Université de Montréal

\section{ISSN}

1188-7109 (imprimé)

1492-1413 (numérique)

Découvrir la revue

\section{Citer cet article}

Malherbe, J.-F. (1993). La théologie dans le champ spéculatif. Théologiques, 1(1), 101-122. https://doi.org/10.7202/602384ar

\section{Résumé de l'article}

Dans la culture actuelle, la théologie souffre d'un discrédit qui procède de la fascination générale à l'égard de disciplines opératoires privilégiées par un monde universitaire devenu largement scientiste. La théologie elle-même entretient, sans doute involontairement, ce discrédit à partir d'une image de soi négative. Or, cette situation est totalement injustifiée aux yeux de la raison philosophique qui, à travers la mise en oeuvre de l'analyse logique des langages spéculatifs, souligne la parenté très profonde qui existe entre théologie, philosophie et sciences opératoires. De reconnaître la validité de cette perspective, la théologie posséderait toute la vis medicatrix naturae pour se remettre en accord avec elle-même et retrouver sa dignité dans le concert des disciplines universitaires. 


\section{L@ i็น

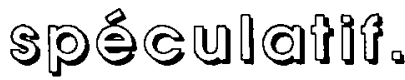

Jean-François MALHERBE

Faculté de théologie Université de Montréal

\section{RÉSUMÉ}

Dans la culture actuelle, la théologie souffre d'un discrédit qui procède de la fascination générale à l'égard de disciplines opératoires privilégiées par un monde universitaire devenu largement scientiste. La théologie elle-même entretient, sans doute involontairement, ce discrédit à partir d'une image de soi négative. Or, cette situation est totalement injustifiée aux yeux de la raison philosophique qui, à travers la mise en oeuvre de l'analyse logique des langages spéculatifs, souligne la parenté très profonde qui existe er.tre théologie, philosophie et sciences opératoires. De reconnaître la validité de cette perspective, la théologie possèderait toute la vis medicatrix naturae pour se remettre en accord avec elle-même et retrouver sa dignité dans le concert des disciplines universitaires.

Depuis le début des années soixante, la théologie a changé considérablement de figure. Elle a été détrônée et n'est plus reconnue comme la "reine des sciences" par le monde universitaire. Au contraire, le développement des techno-sciences l'a confinée dans le domaine du "non scientifique". Curieusement, il semble que le mouvement conciliaire ait indirectement précipité les choses en soulignant les racines existentielles et historiques de toute théologie authentique. Cette tendance s'est accentuée dans la mouvance herméneutique des travaux théologiques élaborés sous l'impulsion de pensées comme celles de Martin Heidegger, Rudolf Bultmann et Hans-Georg Gadamer. Enfin, 
l'avènement des sciences de l'humain, en mettant en question les catégories fondamentales des philosophies de l'existence, liberté, projet, sens, ont proclamé la mort de Dieu puis celle de l'homme et renforcent ainsi la tendance à la sécularisation des discours universitaires prenant pour objet le phénomène religieux.

En définitive, comme je l'ai souligné à la suite de Jean Ladrière1, la théologie se trouve reléguée aujourd'hui, à tout le moins dans le monde universitaire, dans un statut d'infériorité implicite. Et il convient de reconnaître qu'elle souffre de cette intimidation. Certains voient dans l'asthénie théologique, dont nous pouvons observer chaque jour les symptômes dans nos Facultés, le signe avant-coureur de son agonie. Pour ma part, je pense plutôt que cette pathologie procède d'une image de soi négative, produite par la fascination à l'égard de disciplines opératoires privilégiées par un monde universitaire devenu largement scientiste, et que la théologie possède toute la vis medicatrix naturae pour se remettre en accord avec elle-même 2.

Face à ce diagnostic, une thérapie se recommande d'elle-même: décoder d'abord l'asthénie susmentionnée considérée comme expression d'une crise d'identité de la théologie; déconstruire ensuite le pouvoir de fascination du carcan imaginaire dans lequel la théologie s'est laissé emprisonner -sans doute pour survivre dans un environnement hostile. II s'agit donc de laisser se dénouer les anciennes entraves et d'ouvrir le champ à de nouvelles configurations susceptibles de favoriser une plus grande justesse de l'autocompréhension de la théologie dans le champ interdisciplinaire de l'Université.

La déconstruction de l'asthénie théologique suppose un instrument d'analyse: j'ai choisi de privilégier ici l'analyse du langage qui, non seulement m'est assez familière, mais paraît susceptible d'éclairer notre question d'un jour particulièrement révélateur. Je mènerai mon travail en trois étapes. Dans la première, je planterai l'arrière-fond théorique de l'analyse. Dans la seconde, je tenterai de spécifier les modalités particulières des sciences, des philosophies et des théologies dans le champ des discours spéculatifs. Dans la dernière, je soulignerai la pertinence particulière de la théologie parmi les

1 L'articulation du sens. (2 vol.). Paris, Cerf, 1984.

2 Le langage théologique à l'âge de la science. Paris, Cerf, 1985. 
langages spéculatifs et sa contribution, à mon sens essentielle, à la construction d'un avenir de la pensée.

De nos jours, les langages "spéculatifs" ont mauvaise presse. Notre culture préfère les langages directement impliqués dans l'action. L'efficacité technique de l'ingénieur, du médecin, de l'homme d'affaire et de l'administrateur l'emporte sur la spéculation du physicien théricien, du philosophe et du théologien. Cette perception des choses at-elle quelque fondement ou bien n'est-elle que le simple préjugé d'une époque fascinée par les prouesses techniques au point de confier aux seuls techniciens la gestion de son avenir, en croyant pouvoir faire l'économie d'une interrogation radicale sur la signification de leurs exploits?

Pour ma part, je pense qu'il s'agit là d'un préjugé qui, pour tenace qu'il soit, reste privé de tout fondement critique. C'est en tout cas ce que fait apparaître une analyse de la question en termes de langage. Peut-on, du point de vue d'une analyse du langage, établir une distinction tranchée entre les langages de l'action et des langages purement spéculatifs, les premiers étant censément en prise directe sur la transformation objective de la réalité, les seconds étant dépourvus de toute référence non subjective à la réalité?

\section{Eléments d'analyse philosophique du langage}

L'une des premières contributions de l'analyse philosophique du langage est de préciser de façon assez claire la distinction entre les langages animaux et la parole humaine. Cette différence nous importe ici au plus haut point car chacun conviendra certainement que les animaux ne pratiquent pas la théologie tandis que les humains s'y adonnent parfois, même si cette pratique du langage n'est pas la mieux appréciée aujourd'hui.

\subsection{Langages animaux et parole humaine}

J'évoque ici le langage métaphorique pour souligner la différence entre les langages animaux et les langages humains. Les espèces animales possèdent des langages symboliques. Je pense par exemple au langage des abeilles qui utilisent le symbolisme géométrique des coordonnées polaires pour indiquer la position d'un point de leur 
territoire par rapport à la ruche et au soleil. Mais les langages animaux sont stéréotypés et stables, ils ne connaissent pas d'étymologie. Les humains, par contre, sont des créateurs de métaphores et leurs langages sont toujours en évolution. Les sens qu'ils permettent d'exprimer s'ancrent dans une histoire marquée par des récits traditionnels et des oeuvres littéraires. II n'y a pas d'humains sans tradition ni héritage.

Aristote définissait la vérité comme le fait de dire de ce qui est qu'il est, et de ce qui n'est pas qu'il n'est pas. Et le mensonge comme le fait de dire de ce qui est qu'il n'est pas, et de ce qui n'est pas qu'il est. Cette définition est précieuse et nul n'en contestera le bien fondé. Mais l'être humain possède cette faculté de dire sans mentir de ce qui n'est pas qu'il est.

Ainsi, par exemple, lorsque nous disons avec Shakespeare que "le temps est un mendiant", nous disons de ce qui n'est pas (le temps n'est pas un mendiant) qu'il est. Et cependant nous ne mentons pas. Nous opérons simplement ce que Paul Ricoeur appelle une "torsion métaphorique ${ }^{n 3}$. L'être humain a cette faculté de parler "comme si", c'est- à-dire d'affirmer à la fois que le temps n'est pas un mendiant et qu'il est un mendiant. Ce processus de métaphorisation est à la base même de la signifiance du langage, c'est-à-dire du dynamisme sémantique qui nous ouvre l'histoire de notre propre signification d'êtres humains.

Les humains sont des animaux qui ne sont pas que des animaux. Les humains sont des animaux parce qu'ils partagent avec ces derniers les fonctions biologiques qui les caractérisent, notamment se nourrir, s'abriter et copuler. Mais les êtres humains ne sont pas que des animaux. Ils possèdent en effet cette faculté surprenante d'engager leur fonctionnement organique dans une signifiance qui leur est propre.

Les humains ne se contentent pas de s'abriter, ils habitent. Ils s'investissent personnellement et historiquement dans une habitation. Ils ont même développé un art qui préside à la construction de leurs habitations: l'architecture. Les humains ne se contentent pas non plus de se nourrir, ils aiment à déguster et ils ont inventé un art de la table, une gastronomie qui leur est propre. Les êtres humains ne se contentent pas de copuler, ils veulent aimer et être aimés et ils ont élaboré également

${ }^{3}$ La métaphore vive. Paris, Seuil, 1975. 
des arts de l'amour ou arts érotiques auxquels il ne semble pas que les animaux aient accès.

Quelle différence y a-t-il entre s'abriter et habiter, entre se nourrir et déguster, entre copuler et aimer? La différence tient essentiellement à la signification portée par les gestes, signification qui émerge de la parole échangée entre habitants, entre commensaux ou entre amants.

Autre exemple: si nous disons d'un professeur qu'il n'est pas un aigle, nous n'énonçons pas seulement une banalité qui n'aurait de sens qu'en zoologie. Nous disons aussi que ce professeur ne voit pas les choses d'assez haut ni d'un regard suffisamment aigu. Nous disons qu'il a le nez sur son sujet et le situe péniblement dans un ensemble plus vaste. Mais en disant d'un professeur qu'il n'est pas un aigle, nous laissons entendre qu'il aurait pu l'être. Ce dernier énoncé, manifestement faux au plan de la biologie, exprime d'ailleurs très bien ce que nous voulions insinuer en réalité. Nous pourrions dire aussi d'un professeur assis dans sa chaire qu'il tombe de haut même s'il reste immobile. Cela peut signifier qu'il se prenait à tort pour un aigle alors qu'en fait il n'est qu'une simple taupe.

Notre parole joue sur les mots pour dire ce que, prise au pied de la lettre, elle ne dit pas. La parole humaine est métaphorique. Elle s'appuie sur elle-même pour se porter plus loin. Elle dit ce qui n'est pas (que tel professeur est un aigle) pour dire ce qui est (que ce professeur s'est fait remarquer par la hauteur et la pénétration de ses vues).

C'est cette propriété des langages humains qui leur ouvre la voie vers la spéculation, vers un "faire voir $x$ comme un $y^{n}$.

\subsection{L'implication du sujet dans sa parole}

Si nous jouons sur et avec les mots, si nous pouvons opérer des "torsions métaphoriques", c'est parce qu'à travers notre langue maternelle nous sommes les héritiers d'une tradition et d'une manière, plus ou moins unifiée ou éclatée, de considérer le monde, les autres et nous-mêmes. Pas de métaphore, en effet, sans appui sur un sens premier. Et qu'est-ce qu'un sens premier sinon, comme dit Paul Ricoeur, un sens métaphorique usé et oublié. Nos langages ont une histoire. Il sont la couche apparente d'une longue sédimentation que nous appelons l'étymologie. Cette remarque suffirait à démontrer le caractère 
insurpassablement subjectif de toute parole humaine. Mais it n'est sans doute pas inutile de reprendre la question à partir de la théorie des performatifs.

Selon cette approche, qui s'enracine chez le second Wittgenstein au travers des travaux de John L. Austin, John Searle et Donald D. Evans ${ }^{4}$, tout énoncé du langage humain est marqué par une "force illocutionnaire" que l'on peut représenter symboliquement par un opérateur indiquant le type d'action que cette énonciation effectue lorsqu'elle est proférée.

Ainsi, pour prendre un exemple vraiment schématique, l'énoncé $e=$ (Jean fume beaucoup)

doit-t-il être marqué par un "opérateur phatique" $\mathbf{P h}$ pour devenir une énonciation E. On appelle "opérateur phatique" un marqueur indiquant le type d'usage que le locuteur fait d'un énoncé dans une énonciation:

Ph $(e)=E$

- donner un ordre:

IMPERATIF $\quad(\mathbf{e})$ = Fume beaucoup, Jean!

- formuler une interdiction:

INTERDIT

(e) = Jean, je t'interdis de beaucoup fumer!

- poser une question:

QUESTION $(\mathbf{e})$ = Jean fume-t-il beaucoup?

Jean, fumes-tu beaucoup?

- décrire un fait:
DESCRIPTION
(e) = Jean fume beaucoup.

Mais l'énoncé e s'analyse lui-même en deux parties: ce dont on parle et ce qu'on en dit: la référence et la qualification.

$$
e=(R, Q)
$$

Dans le cas qui nous occupe, la référence est la personne répondant au prénom Jean et la qualification est de consommer une grande quantité de tabac

4 Wittgenstein, L. Philosophische Untersuchungen - Philosophical Investigations. Oxford, B. Blackwell, 1953. Austin, J.L. How to do Things with Words? Oxford, Oxford Univ. Press, 1963; Evans D.D. The Logic of Self-involvement. New-York, Herder, 1969. 
Mais, finalement, un locuteur parle de quelqu'un ou de quelque chose dans un contexte particulier, et à quelqu'un d'autre, à un auditeur, un interlocuteur (que l'on appelle parfois en analyse du langage un allocutaire). Par conséquent (si Cont symbolise le contexte, Loc le locuteur, celui qui parle et Alloc l'allocutaire à qui il parle), la formule complète d'un acte de langage se lit comme suit:

Cont < Loc : Ph ( R, Q) : Alloc >

Cela signifie qu'un acte de langage, ou une "prlse de parole" est un acte par lequel un lecuteur intervient dans un certain centexte pour communiquer à un allocutaire le rapport qu'il établit entre ce dont il parle et ce qu'il en dit.

Cela nous apporte un second enseignement: la parole humaine est non seulement métaphorique, mais encore "performative"; elle effectue quelque chose par le simple fait d'être prononcée: donner un ordre, poser une question, promulguer une interdiction, décrire un fait. Aucune parole humaine, aucune énonciation de langage ne fait exception: le locuteur fait toujours quelque chose en disant $c e$ qu'il dit.

Mais une troisième leçon peut être tirée des considérations qui précèdent. Elle concerne le caractère de réciprocité de toute parole humaine. II n'y a pas de "je" sans "tu", ni de "tu" sans "je". Et il n'y a pas de "il" sans "je" ni sans "tu". Nous pouvons prendre à tour de rôle la position "je", la position "fu" ou la position "il" dans le dialogue, mais ces positions n'existent pas séparément; elles n'ont de sens que les unes par rapport aux autres. Nous sommes toujours déjà insérés dans une structure de réciprocité. C'est sans doute là le fait humain le plus fondamental et l'on pourrait développer toute son importance dans la question de l'éthique. Mais ce n'est pas le thème de la présente intervention.

L'appartenance de la parole à la sphère de l'action se manifestait déjà de façon extrêmement claire dans le processus de métaphorisation auquel j'ai déjà fait allusion. Une métaphore effectue, en effet, une espèce de torsion métaphorique qui fait voir ce dont il s'agit sous un jour inédit. La contradiction implicite que contient toute métaphore, dire de ce qui est qu'il est et en même temps qu'il n'est pas, dire du mal qu'il a une racine alors que tout un chacun sait parfaitement qu'il n'en a pas, c'est effectuer une transformation du référent (ce dont on parle) qui le fait apparaître comme quelque chose qui a une racine c'est-à-dire comme quelque chose qui peut être extirpé de l'endroit où il apparaît. 
Créer des métaphores et en user jusqu'à les user, telle est la pratique du langage. Cette pratique est déjà, comme son nom l'indique, une action, mais elle l'est aussi lorsqu'on la considère sous l'angle de ses effets puisqu'elle nous fait voir les choses dont elle parle sous un jour qui les rendra intelligibles ou confuses, transformables ou immuables, déplaçables ou immobilisées.

C'est en définitive tout notre rapport aux choses, au monde, à autrui et à nous-mêmes qui est marqué par la parole. On pourrait même dire que c'est la parole qui nous ménage un accès à ces différentes réalités en nous les présentant sous tel ou tel jour, dans tel ou tel contexte, selon tel ou tel projet. La parole rend possibles certaines actions et en fait apparaître d'autres comme vouées à l'échec depuis le début.

\subsection{La parole et l'action}

II n'y a pas de parole sans action mais il n'y a pas non plus d'action sans parole. Pour reprendre l'exemple schématique du sculpteur, on pourrait dire que l'artiste n'aurait absolument aucune possibilité d'agir s'il n'était déjà inséré dans un réseau de communication par les paroles qui lui ont été adressées et celles qu'il a lui-même proférées. Comment disposerait-il de marbre s'il ne s'était adressé luimême à un carrier ou à un intermédiaire? Comment aurait-il en tête un modèle s'il n'était en relation avec quelqu'un qui lui en a fourni le thème, l'occasion ou l'idée? Comment entreprendrait-il son travail s'il n'avait appris de l'exemple commenté d'un maître comment manier le marteau et le burin? Comment aurait-il pu avoir simplement l'idée d'entreprendre son travail s'il n'avait pu lui concevoir un but qui, même s'il devait rester implicite, est tributaire de la vision du monde, de la société et de sa propre vocation qui est véhiculée par le langage ordinaire qu'il a hérité de son milieu en même temps que son existence ?

La manipulation d'outils suppose la parole. En effet, le fait même que des outils existent suppose qu'ils aient été mis au point par la collaboration de leurs utilisateurs et cette collaboration, ne fût-ce que par la division ou la coordination qu'elle implique, suppose les recours à la parole.

D'autre part, la manipulation d'un objet suppose que cet objet ait été désigné dans le langage par des expressions appropriées, sinon comment le manipulateur pourrait-il l'isoler du contexte? Ce qui sépare 
un objet de son contexte, c'est la façon dont il est mis en scène dans le langage. C'est un travail langagier analogue qui construit la distinction entre le trait signifiant et la tache d'encre. Une illustration frappante de ce fait est la nécessité où se trouve le profane d'apprendre à voir ce qui se passe sous l'objectif d'un microscope ou sur l'écran cathodique d'un appareil à échographie. Si personne ne lui explique comment départager les traits significatifs des autres, jamais il ne parviendra à identifier les différents composants d'une cellule ou la structure anatomique de l'embryon qu'il a pourtant littéralement sous les yeux.

La parole et l'action s'impliquent donc mutuellement et chacune déborde l'autre. En effet, la parole anticipe l'action dans le calcul d'un plan ou d'une stratégie, et elle la prolonge dans le compte rendu, l'échange de souvenirs ou la méditation. Tandis que l'action de son côté, par ses multiples aspects involontaires, inconscients ou automatiques, échappe toujours en partie à la parole qu'elle accomplit ou que bien souvent elle précède, comme dans la situation de réflexion après l'échec. L'action est le milieu de la parole et la parole est le ciment de l'action. Elles sont distinctes, assurément, mais elles s'imprègnent mutuellement au point qu'il n'est pas possible de les dissocier.

La parole dépourvue de tout contexte d'action serait également dépourvue de tout sens. Et l'action sans contexte de parole ne serait pas une action. Elle serait une simple accumulation d'actes sans aucun autre lien entre eux que leur succession temporelle et le fait qu'ils soient effectués par le même agent. Mais la notion même de l'identité de cet agent aurait-elle un sens si celui-ci ne bénéficiait pas de la stabilité existentielle que lui confère la parole de ses semblables l'appelant par son propre nom?

L'existence d'un être humain est une trajectoire d'actes. Parmi ces actes, certains sont des actes de langage, des paroles échangées. Les actes de langage sont autant de points où la trajectoire d'un être humain entrecroise celles de ses semblables. Les actes de langage forment donc la trame du tissu social sur laquelle chaque être humain inscrit le dessin de sa propre destinée. Ce sont eux qui confèrent à notre existence le sens que nous lui donnons.

Mais revenons plus directement à notre préoccupation première. Toutes les remarques accumulées jusqu'ici tendent vers une même conclusion: tous les usages humains du langage sont marqués par le recours à des métaphores et une intention de communiquer quelque chose 
à queliju'un. Une parole humaine fait voir quelque chose à quelqu'un d'une certaine façon dans un contexte particulier. Je puis vous faire voir Jean comme un gros fumeur ou comme quelqu'un à qui j'ai interdit de fumer. Bref,

\section{faire voir $x$ comme $y$}

est le mouvement fondamental de tout langage humain. Mais ce mouvement, qu'est-il d'autre que celui de la spéculation? "Spéculer", qu'est-ce d'autre, en effet, que d'être pour ainsi dire un visionnaire? "Speculare" signifie voir. Un "speculum" est un instrument que les médecins utilisent parfois pour voir à l'intérieur du corps.

C'est donc dire que toute parole humaine est spéculative et, par conséquent, que le langage des différentes disciplines universitaires qui forment l'objet de notre préoccupation, sont des langages spéculatifs. Ils se distinguent cependant entre eux par la manière dont ils construisent leurs façons de voir, les spéculations qui leur sont propres.

\section{2 "L'air de famille" commun aux sciences, à la philosophie et à la théologie}

\subsection{Les langages scientifiques}

Si vous le permettez, j'aimerais examiner le cas particulier des sciences biomédicales. D'abord parce que leur langage m'est plus familier que d'autres. Ensuite, parce que chacun de nous finit toujours par avoir affaire avec les spécialistes qui parlent ce langage. Et, enfin, parce que c'est dans le domaine médical que l'articulation de l'opérativité techno-scientifique et des questions du sens de l'existence humaine et de la finalité des savoirs opératoires se posent de la façon la plus aiguë.

Les sciences biomédicales qui sous-tendent toute la problématique de la médecine contemporaine sont une vaste entreprise destinée à diminuer la marge d'incertitude de la décision médicale en fournissant au médecin des connaissances nouvelles, plus précises et plus fiables au sujet des réalités auxquelles il est confronté. A première vue, ces sciences sont donc de précieuses auxiliaires de soins de meilleure qualité au bénéfice des malades. Cependant, la mise en oeuvre 
de ces sciences peut produire des "effets pervers" 5 (qu'il appartient à l'éthique de prévenir, mais que je ne discuterai pas ici puisque ce n'est pas mon objet). En effet, ces sciences envisagent toujours leur objet sous les traits d'un système. Elles effectuent une réduction de l'humain au système organique qui en constitue le support matériel. Les médecins sont ainsi toujours tentés d'abstraire de la personne humaine singulière, la machine cybernétique dont ils se font les ingénieurs.

\subsubsection{Une réduction épistémologiquement nécessaire ...}

Selon cette approche méthodologique, l'humain et ses parties sont considérés comme isolables de leur contexte, c'est-à-dire notamment des systèmes avec lesquels ils se trouvent en interaction. Ainsi, par exemple, le système circulatoire est considéré comme séparé, distinct, différent du système respiratoire ou du système nerveux. Il est aussi considéré comme décomposable en sous-systèmes. Ainsi le sang, par exemple, peut-il être considéré comme un système indépendamment du coeur. Mais tous deux s'emboîtent comme des soussystèmes dans le système respiratoire. Par rapport au sang, les globules rouges, par exemple forment également un sous-système.

C'est par hypothèse méthodologique que l'on considère qu'un système est toujours emboîté dans un autre et qu'inversement d'autres viennent s'emboîter en lui. Un système est encore considéré comme évoluant au cours du temps. Le système circulatoire se développe au cours de l'embryogenèse et se détériore au cours du vieillissement de l'organisme ou de certaines maladies particulières comme l'artériosclérose ou l'infarctus du myocarde. Mais chaque étape de l'évolution d'un système peut être caractérisée par un état. L'état d'un système à un moment donné comprend toute l'information dont on dispose à son sujet à l'instant considéré. La mesure de la pression artérielle ou du pouls, par exemple, sont des éléments de l'état du système circulatoire de tel organisme à tel moment.

Etudier un système consiste à le décrire en ses moments successifs et à tenter de formuler les lois auxquelles obéit son évolution

5 Cf. mon ouvrage Pour une éthique de la médecine. Paris, Larousse, 1987. C'est la raison pour laquelle l'éthique exige le consentement libre et éclairé des sujets de recherche, dans l'espoir que l'inévitable suspension de l'humain qu'impose la méthodologie scientifique ne soit pas le dernier mot et que le savoir ainsi acquis soit approprié au service de ceux et celles qui ont permis de le constituer. 
au cours du temps. Si ces dernières sont correctement énoncées, elles permettent de prévoir l'évolution du système en fonction des paramètres qui, à chaque instant, déterminent cet état. Sans cet ensemble d'hypothèses méthodologiques, il serait impossible de connaître le fonctionnement d'un système ni d'interagir avec lui de façon efficace.

En considérant l'humain comme un système, les sciences biomédicales, et la physiopathologie en particulier, se sont ménagé la voie de l'opération puisqu'elles peuvent en prévoir l'évolution en fonction de la variation des paramètres qui le définissent. Cette conquête méthodologique s'est avérée d'une portée considérable qu'il n'entre pas dans mon intention de dénigrer. Mais l'opérativité ainsi acquise se paie à grands frais, car elle a réduit l'humain à une machine cybernétique. Elle l'a abstrait de son histoire particulière, de sa subjectivité propre, de son existence vécue.

L'objectivation de l'être humain par les sciences biomédicales consiste à mettre entre parenthèses certains aspects du sujet pour en mettre d'autres en évidence. Le temps chronométrable est abstrait de la durée; la géométrie, de l'espace qualitatif; l'entropie, du vieillissement; l'organisme, de la personne; le métabolisme biologique, de l'existence personnelle. Bref, les sciences biomédicales découpent la chair humaine pour en extraire une abstraction nommée machine cybernétique. L'objectivité de l'observation est mise en évidence au prix de la mise entre parenthèses de l'auto-compréhension du sujet. Les déterminismes communs sont abstraits de l'exercice concret des libertés personnelles.

C'est dire que, du point de vue de l'analyse philosophique adopté ici, le langage scientifique se caractérise par trois traits particuliers:

1. Tout d'abord, les réseaux de métaphores qu'il se construit pour "spéculer", pour voir les choses de "façon scientifique" sont artlculés par une logique de type mathématique et quantitatif (dans le cas de la médecine: trop de cholestérol, pas assez de tension, etc).

2. Ensuite, la visée qui marque tout énoncé scientifique obéit à la logique de l'interprétation opératolre. Les sciences se construisent par hypothèses qui sont systématiquement soumises au 
verdict des faits par l'intermédiaire des procédures expérimentales ${ }^{6}$. Finalement les sciences répondent à la question: comment interpréter (faire voir) la réalité matérielle pour qu'elle se laisse façonner en fonction de nos projets d'action?

3. Enfin, du côté de l'opérateur phatique, les disciplines scientifiques visent à la formulation d'énoncés dont le contenu soit aussi indépendant que possible de la subjectivité de l'individu singulier qui les profère. C'est dire qu'elles se sont donné des procédures qui visent, pour ainsi dire, à désimpliquer le sujet des énonciations dont il est l'auteur. $\mathrm{Ce}$ sont $\mathrm{ce}$ qu'on pourrait appeler avec Karl Popper les procédures d'intersubjectivité critique ${ }^{7}$. Chaque énoncé doit pouvoir être vérifié par un autre sujet de même compétence que le premier. Et les énoncés ainsi construits ne seront jamais considérés comme vrais mais seulement comme provisoirement non réfutés. C'est donc du jeu critique des conjectures et des réfutations qu'émergent progressivement des interprétations opératoires de larges pans de la réalité.

En un mot comme en cent, pour revenir aux sciences biomédicales, l'organisme est abstrait de la personne, le corps qu'on a du corps qu'on est, dans la visée de construire une interprétation du corps qui le dispose à l'opération curative. Mais cette visée, si elle perd de vue sa véritable finalité qui est la santé de l'humain, comporte des risques majeurs de l'asservir plutôt que de le servir.

\subsection{2 ...qui risque de dénier la souffrance vécue du sujet}

Les sciences biomédicales, en raison même du caractère opératoire qui est le leur, comportent le risque de négliger la souffrance vécue par le sujet, souffrance dont le soulagement est sa raison d'être. II n'empêche que la parole naît toujours de la souffrance d'un sujet qui chemine à la recherche de sa véritable identité au coeur même de la crise qu'il vit, au coeur même de la déchirure qui le fait crier. Encore faut-il, pour que son cri devienne parole, qu'il soit entendu par autrui pour ce qu'il est 8 .

6 Cf. mon ouvrage Epistémologies anglo-saxonnes. Paris, P.U.F., 1982.

7 Cf. mon ouvrage La philosophie de Karl Popper et le positivisme logique. Paris, P.U.F.,1977, 1979.

8 On peut d'ailleurs se demander si l'objectivation de l'humain par les technosciences biomédicales n'est pas utilisée en grande partie pour protéger les soignants des cris des 
Praticiens de l'objectivation, les soignants - par un étrange retournement de leur méthodologie -, sont censés ne pas souffrir et cela les rend sourds aux cris de ceux qu'ils soignent. Ces cris ne parviennent donc pas à se faire entendre. Leur message est obnubilé par l'expression de la douleur. La douleur du soigné provoque l'action technique du soignant qui tente de l'en débarrasser ${ }^{9}$. Le soigné est transformé en objet de douleur, son cri est aussitôt réduit à l'expression d'une douleur techniquement maîtrisable. Mais la souffrance dont témoigne son cri est considérée implicitement comme méprisable précisément parce qu'elle ne peut être conjurée par un acte technique. Or, ne pas entendre le cri de l'homme souffrant comme étant le cri d'un sujet affronté à la mort dans son désir de vivre, c'est le condamner au silence, l'exclure de la véritable conversation institutrice de notre humanité.

Mais il est bien des manières de refouler la parole qui s'articulerait si la souffrance était entendue pour ce qu'elle est. La plus efficace consiste à considérer que l'être humain n'est pas le sujet d'une telle souffrance ni a fortiori d'une telle prise de parole.

Lorsque l'être humain se réduit à l'image scientiste que notre culture scientifique se forge de lui, il se ment à lui-même en croyant se raconter la vérité. Lorsque la médecine se trouve complice de ce mensonge ou, pire, le cultive, elle renie sa raison d'être et se mue en idéologie mortifère. De ce mensonge, en effet, résulte la mort du sujet, dans le chef du soignant (comme du soigné d'ailleurs). Finalement, et paradoxalement, c'est par le truchement du développement technoscientifique qu'elle cultive, que la société occidentale dénie -et par conséquent précipite- cela même qu'elle cherchait à corriger: la souffrance.

En définitive, ces remarques soulignent le fait que la médecine, qui passe pour l'un des fleurons les plus prestigieux de l'esprit scientifique, est à la fois promotrice et victime du scientisme dans lequel je vois également l'étiologie de l'asthénie théologique.

soignés qui, s'ils les entendaient, les renverraient eux-mêmes aux cris muets de leur propre angoisse.

9 Cf. Vasse, D. Le poids du réel, la souffrance. Paris, Seuil, 1987. 


\subsection{Les langages philosophiques}

Les discours philosophiques, qui visent le développement de l'autonomie des sujets par la mise en oeuvre de cette même autonomie, s'efforcent de laisser transparaître l'intérêt qui les porte. lis sont donc, pour ce motif même, relativement bien appropriés au service de la vérité et à la critique des idéologies scientifiques, religieuses et pseudophilosophiques. Est-ce à dire que les discours philosophiques détiennent la vérité? Nullement, mais il est vraisemblable que c'est parmi eux qu'on pourra trouver quelques uns des moins mauvais chemins vers elle.

Quoi qu'il en soit, les discours philosophiques, lorsqu'ils procèdent à l'élucidation de l'implicite de leurs propres langages, mettent en oeuvre dans leur propre effectuation cela même qu'ils visent: la recherche de l'autonomie 10 .

Du point de vue de l'analyse linguistique, les langages philosophiques manifestent des traits spécifiques qui peuvent se résumer à trois, en correspondance d'ailleurs avec les traits distinctifs des langages scientifiques.

1. Les langages philosophiques consistent à construire des interprétations de l'existence humaine ef de ses conditions de possibilités qui en fassent apparaître le sens (ou le non sens, c'est-àdire l'absence d'un sens que requerrait l'existence humaine). La requête de sens est une requête d'unité; aussi les réseaux de métaphores auxquels la philosophie a recours sont-ils régis, du moins en principe, par le principe de non contradiction. Evidemment, ce principe ne s'applique pas à la lettre des métaphores philosophiques mais à l'esprit dans lequel on y recourt. Pour illustrer ceci, je me reporte simplement à l'image que j'utilisais ci-dessus lorsque je parlais de l'être humain comme d'un animal censé tenir parole, ou lorsque je comparais une existence humaine à une trajectoire dont les points seraient des actes.

2. La visée des interprétations philosophiques, comme je viens de le mentionner, est le sens et le non sens. On pourrait dire des philosophies qu'elles sont des Interprétations sémantiques de la réalité. Evidemment, cela n'exclut pas qu'elles aient éventuellement une

$10 \mathrm{Cf}$. à ce sujet mon ouvrage Le risque préventif, éthique, police et thérapie Paris/Montréal, Cerf/Fides, 1993 ( sous presse) dont la troisième partie propose précisément une éthique philosophique de l'autonomie. 
visée opératoire. Mais leur opérativité sera de type éthique plutôt que technique. Elle visera plutôt à faire apparaître le sens ou le non sens de l'action, ou les conditions de possibilité de ce sens, qu'à organiser techniquement les gestes à poser pour mener à bien cette action.

3 Du côté de l'opérateur phatique, je dirais que les philosophes sont toujours impliqués subjectivement dans leurs discours mais qu'ils se donnent, ou devraient se donner, la discipline de ne rien énoncer qui ne soit universalisable. Leur propos n'est pas la désimplication du sujet à l'égard de son discours, mais l'universalisation du discours: ce que je dis est-il éclairant pour tous mes semblables?

\subsection{Les langages théologiques}

De leur côté, les langages théologiques se caractérisent également par des traits spécifiques. Pour la clarté de l'exposé, je les envisagerai dans le même ordre que celui que j'ai adopté pour les langages scientifiques et philosophiques. Précisons d'emblée que je considère ici les langages théologiques au sens strict de langages visant, de l'intérieur même de la foi, à l'intelligence de la foi.

1. Au niveau de la loglque qui régit les réseaux métaphoriques qu'elles construisent, il n'y a pas, à mon sens de distinction majeure entre la philosophie et la théologie. La distinction se trouve plutôt dans la source de ces métaphores. Evidemment, les théologies peuvent puiser leurs métaphores dans l'ensemble des langages humains. Mais il est manifeste qu'elles ont une prédilection bien marquée pour les métaphores bibliques, patristiques et traditionnelles. Remarquons que ces dernières, si on remonte à leurs plus lointaines origines, s'enracinent toutes dans la vie concrète d'un peuple se sachant élu de Dieu et souvent infidèle à l'Alliance. Mais le vocabulaire de cette simple remarque est déjà la démonstration de ce qu'elle souligne.

2. La visée des interprétations théologiques est double: l'intelligence de la foi à l'intention des croyants, en quoi consiste ce qu'on appelle traditionnellement la théologie dogmatique; et l'intelligence de la foi à l'intention des non croyants, en quoi consiste ce qu'on appelle traditionnellement l'apologétique. Mais quoi qu'il en soit de l'allocutaire visé, la construction d'une théologie suppose l'adhésion au message dont 
elle tente de rendre raison. La théologie consiste toujours en une tentative de rendre raison de ce que l'Église nous propose à croire.

3. C'est dire que l'opérateur phatique du discours théologique est complexe. D'un côté, il marque l'adhésion au message qu'il tente d'expliciter et, de l'autre, il suspend cette adhésion dans la prise de distance critique qu'exige tout essai de rendre raison. Pour souligner cette tension, je dirais volontier que l'opérateur phatique propre aux énonciations théologiques met le locuteur en position d'adhésion suspendue par rapport à l'énoncé dont il vise à rendre raison.

Récapitulons. Science, philosophie et théologie sont toutes trois des langages spéculatifs constitués de métaphores dont les réseaux sont régis par le principe de non contradiction et, dans le cas des sciences, par l'appareil mathématique et quantitatif. La visée des sciences est l'interprétation opératoire de la réalité. Celles de la philosophie et de la théologie sont des interprétations sémantiques. La théologie se distingue toutefois de la philosophie par son rapport primordial à des événements salvifiques et à leurs conséquences dont il s'agit de manifester le sens. Pour ce qui est des opérateurs phatiques, les sciences se caractérisent par la désimplication, la philosophie par l'universalisation et la théologie par l'adhésion suspensive à visée universelle.

\section{Philosophie et théologie}

\subsection{La philosophie et les risques du théologien}

Les considérations qui viennent d'être énoncées indiquent que les théologiens courent deux risques symétriques dans l'exercice de leur art qui consiste à disposer leurs phrases et leurs mots de façon à faire apparaître dans la cohérence du discours un réseau de métaphores capable de structurer l'expérience de foi et d'en renforcer ainsi la vacillante certitude.

Le premier de ces risques est de ne pas prendre, à l'égard de l'expérience personnelle ou communautaire de la foi, une distance suffisante pour pouvoir en rendre raison. C'est souvent faute d'une maîtrise suffisante des langages philosophiques que les théologiens ne parviennent pas à décoler véritablement de l'expérience vécue et à élaborer leur expression de l'intelligence de la foi dans une visée 
suffisante de l'universel. Ce défaut est mineur car, bien qu'il prive l'expérience religieuse de la structuration qui lui conférerait sa véritable dimension kérygmatique, il ne met pas véritablement la foi en péril.

Le second risque courru par les théologiens est bien plus dangereux. II consiste à se laisser prendre au jeu du langage et à croire, par conséquent, épuiser l'expérience de la foi dans des formules qui prennent une distance telle à son égard que le lien vital s'en trouve rompu. La théologie, alors, se fait idéologie d'autant plus sournoisement active d'ailleurs qu'elle se pose en vérité éternelle. La "théologie", lorsqu'elle prend cette voie, loin d'être une salutaire adhésion de foi médiatisée dans une clarifiante suspension de la foi, devient, par la vertu d'une diabolique perversion, mise en scène idolâtre de sa propre pseudo-vérité, refoulant dans l'ombre la vérité de son imposture. C'est le risque de prévarication, le risque de collusion avec la partie adverse, qui forme la menace majeure pesant sur l'art du théologien.

Selon le mot de Jean Ladrière: son expression dans le langage achève l'expérience, c'est-à-dire à la fois la porte à son point culminant et la détruit. En effet, l'expérience qui ne vient pas au langage s'estompe et disparaît de la mémoire. Tandis que l'expérience qui vient au langage vise à l'universel et peut s'épanouir jusque dans la communication. Mais lorsqu'elle est coulée dans des phrases, si justes soient-elles, l'expérience se trouve dénaturée. Les mots qui la disent la décrochent, pour ainsi dire, de son état natif. Mais sans les mots, l'expérience s'enliserait dans les sables de l'oubli. Tout l'art du théologien est donc d'éviter les deux risques qui pèsent perpétuellement sur son travail. Sa meilleure garantie sera sa participation active à la vie des communautés de foi et sa capacité de dégager philosophiquement la portée universelle de toute expérience singulière.

\subsection{Raison et révélation sont-elles en concurrence?}

Dans mon travail, je tiens très fort à la distinction entre philosophie et théologie. En théologie, on part de la Révélation de Dieu en Jésus-Christ, on part toujours de la conviction que Jésus-Christ, vrai Dieu et vrai homme, nous révèle Dieu et le plan d'amour qu'il a formé à notre sujet. En philosophie, par contre, on ne présuppose aucune révélation. 
En philosophie, l'être humain s'appuie sur ses propres forces pour tâcher de comprendre qui il est et quelle est sa destinée. Des chrétiens qui s'exercent à la philosophie tentent de construire une interprétation de l'existence aussi indépendante que possible de leur foi. Evidemment, cette indépendance n'est jamais totale car 'sute philosophie s'enracine dans une tradition. Aucune d'entre elles n'a jamais été capable d'opérer une véritable "tabula rasa" à partir de laquelle tout recommencerait à zéro. Toute philosophie est reprise critique d'une culture et d'une (ou de plusieurs) tradition(s).

II n'est donc pas anormal, aux yeux mêmes de l'exigence philosophique la plus critique, que des chrétiens partent d'une culture imprégnée de christianisme pour construire leur philosophie. Simplement, en tant que philosophes, ils considéreront cette culture comme un héritage social sans préjuger que tous ceux qui s'en nourrissent et $y$ contribuent ratifient personnellement la foi religieuse dans laquelle elle s'enracine.

II n'est donc pas du tout anti-philosophique de prétendre construire une philosophie qui tente de recueillir le meilleur de ce que l'humain a pu découvrir de lui-même depuis des millénaires dans le contexte d'une foi religieuse. II n'est d'ailleurs pas non plus antiphilosophique d'être engagé personnellement dans une foi religieuse pour autant qu'on soit attentif à ne pas confondre philosophie et théologie.

Je pars donc en philosophie d'une conception de l'humain pétrie de christianisme et d'éléments de la grande tradition du judaïsme, mais à la différence des théologiens, j'accepte que n'importe quel énoncé venant de cette tradition puisse être mis en question.

Aux yeux du croyant, cette attitude critique -sceptique diront sans doute certains- s'enracine dans un acte de foi: elle trouve sa justification non seulement dans la méthode philosophique mais encore dans la foi chrétienne aux yeux de laquelle l'exercice le plus critique de la philosophie est aussi une nécessité. Cette déclaration peut paraître étrange. Elle n'en est pas moins justifiée.

Le christianisme nous propose de croire que Dieu en créant l'univers a aussi créé notre intelligence critique comme notre intelligence technique. "Critique", entendu au sens étymologique, veut dire "relatif au discernement". La critique sépare ce qui ne doit pas être mélangé. Etre critique, c'est s'efforcer d'avoir les idées claires. 
"Technique", entendu aussi au sens étymologique, signifie l'"art", la "manière", le "tournemain" bref: le "savoir faire". Aux yeux de la foi chrétienne, notre intelligence critique comme notre intelligence technique sont des dons de Dieu créateur. Mais il est évident qu'au plan humain, l'intelligence technique, faculté par laquelle nous inventons les moyens de réaliser nos fins, est subordonnée à l'intelligence critique qui s'efforce de discerner les fins les plus authentiquement humaines de l'humain.

Le Créateur nous donne donc l'intelligence. Mais II se révèle également en Jésus-Christ. Comme II ne peut ni se tromper ni nous tromper, ce qu'll nous donne dans sa Révélation ne peut contredire ce qu'll nous permet de découvrir par l'exercice authentique de notre intelligence critique. Toute la question est donc de définir l'usage "authentique" de notre intelligence. Mais cela aussi est l'oeuvre de l'intelligence critique: découvrir ce qu'il y a de plus humain dans l'humain. Mais qui d'entre nous prétendrait avoir compris toute la Révélation de façon transparente, claire et définitive? Et, d'autre part, qui pourrait prétendre à l'authenticité absolue de l'usage qu'il fait de son intelligence critique?

Autrement dit, les incompatibilités qui peuvent apparaître -et qui apparaissent relativement fréquemment- entre ce que les croyants comprennent de la Révélation et ce qu'ils comprennent par l'exercice de leur intelligence critique indiquent que quelque chose n'a pas été correctement entendu. Mais cela ne signifie nullement a priori que la Révélation doive prendre le pas sur l'intelligence critique. Les théologiens pourraient avoir mal interprété tel ou tel élément de la Révélation aussi bien que nous pourrions avoir manqué de sens critique authentique dans l'exercice de notre intelligence.

La foi en Dieu Créateur va jusqu'à dire qu'il n'y a pas de contradiction possible, du moins dans l'eschatologie, entre ce que nous pouvons apprendre par l'exercice de notre seule intelligence et ce que nous pouvons apprendre par la Révélation. Je n'ai pas dit que nous pourrions découvrir toute la vérité à partir de nos seuls moyens humains. Mais je pense que ce que nous pouvons découvrir par nousmêmes, si nous nous respectons nous-mêmes dans cette recherche, ne peut pas contredire définitivement ce que nous pourrions apprendre à connaître par la Révélation. 


\subsection{Ulysse et Abraham}

La question des rapports entre philosophie et théologie, abordée ici selon l'angle très restreint de l'analyse du langage, resterait par trop unilatérale si je ne l'envisageais que dans le sens d'une contibution de la philosophie à la théologie. Je ne suis pas un avocat de la philosophia ancilla theologiae!

Je pense que les philosophes, dans l'exercice de leur art, courrent également le risque de se laisser porter davantage par le langage que par la vie et que leur participation attentive à la vie de la Cité est la garantie la meilleure (bien que non absolue, évidemment) de la pertinence de leurs élaborations.

Mais il me semble que l'on peut aller plus loin et parler d'une contribution de la théologie à la philosophie.

En effet, la philosophie entend s'appuyer sur les seules forces de la raison pour tenter d'élucider le sens (ou le non sens) de l'existence humaine. C'est dire qu'elle court le risque de s'enfermer dans la raison en refusant toute considération à ce qui ne serait pas intégrable par la raison. On pourrait dire que la philosophie est à la théologie dans le même rapport qu'Ulysse à Abraham. Ulysse part d'lthaque et y revient après avoir bouclé son tour du monde. La seule chose qui a changé (en plus de la longueur de l'écharpe de Pénélope) est l'expérience qu'il possède de la vie et de ses semblables. Ce n'est pas peu de choses. Mais on reste frappé par le caractère circulaire de son itinéraire. Abraham, par contre se met en route avec pour seule certitude qu'il ne reviendra jamais à son point de départ Le rapport à l'altérité est différent dans les deux cas. Immanente dans le cas d'Ulysse, l'altérité se présente sous les traits de la transcendance dans l'expérience d'Abraham.

Je ne veux pas indiquer par cette remarque que la philosophie serait nécessairement narcissique et la théologie assurément réceptive, mais simplement que philosophes et théologiens ont tout intérêt à se fréquenter et à confronter leurs discours.

\section{Conclusion}

En guise de conclusion, je voudrais souligner que la philosophie et la théologie sont les cousines, sinon les soeurs, des sciences et qu'elles 
appartiennent toutes deux à la famille des langages spéculatifs régis par le principe de non contradiction.

II n'y a donc aucune raison qu'elles se plient à l'intimidation scientiste dont elles sont les victimes en raison du caractère immatériel de leur opérativité.

J'irais même jusqu'à dire que philosophie et théologie sont plus que jamais nécessaires et que leur nécessité apparaît au coeur même des démarches scientifiques qui, chaque fois qu'elles laissent dans l'oubli ou, pire, refoulent la question de leur finalité, se trouvent asservir les humains plutôt que de les servir. 